\title{
ENSAMBLAJE DE MURCIÉLAGOS (MAMMALIA: CHIROPTERA) EN DOS ZONAS DEL DEPARTAMENTO DE SUCRE, COLOMBIA
}

\author{
Adrián Alonso DURÁN ${ }^{1,2}$ y Silfredo Canchila PÉREZ ${ }^{1}$ \\ ${ }^{1}$ Grupo de Investigación en Zoología y Ecología Universidad de Sucre (GIZEUS). ${ }^{2}$ Estadística y Modelamiento \\ Matemático Aplicado a la Calidad Educativa. Universidad de Sucre, Cra 28 \# 5267 Barrio Puerta Roja, \\ Sincelejo-Sucre, Colombia.<adurandelaossa@gmail.com>
}

Recibido: 26/09/2014; aceptado: 25/06/2015

Durán, A. A. y Pérez, S. C. 2015. Ensamblaje de murciélagos (Mammalia: Chiroptera) en dos zonas del Departamento de Sucre, Colombia. Acta Zoológica Mexicana (n. s.), 31(3): 358-366.

RESUMEN. El objetivo de este trabajo fue determinar la estructura y composición de los ensamblajes de murciélagos en los alrededores de la Estación Meteorológica Primates (Colosó) y finca "La Flojera" (Sincelejo), en el departamento de Sucre, Colombia; desde junio de 2011 hasta marzo de 2012. La captura de los murciélagos se realizó con redes de niebla, activas entre las 18:00 a 24:00 h. Se capturaron 427 murciélagos pertenecientes a 26 especies, 17 géneros, seis subfamilias y cuatro familias taxonómicas. La familia Phyllostomidae fue la de mayor diversidad, con 20 especies y el 97.9\% de todos los individuos capturados. Artibeus planirostris fue la especie más abundante con 139 individuos, luego Phyllostomus discolor con 56, Desmodus rotundus y Sturnira lilium, ambas con 37 individuos. De acuerdo con los estimadores de riqueza utilizados, la eficiencia del muestreo osciló entre un 80 y $90 \%$. Al considerar las categorías tróficas, los murciélagos frugívoros presentaron la mayor riqueza de especies (42.3\%). El valor de diversidad de Shannon-Wiener (H') obtenido para la zona 1 (Colosó) fue de 2.4 y para la zona 2 (Sincelejo), de 2.0. La dominancia presentó su mayor valor (0.85) en la estación Primates. El análisis de similitud entre comunidades a través del índice cualitativo de Jaccard fue de 0.53 . Las diferencias encontradas en ambas zonas pueden estar asociadas a las diferencias en la vegetación.

Palabras clave: Artibeus planirostris, ensamblaje, murciélagos, Sucre.

\section{INTRODUCCIÓN}

En la actualidad el Bosque seco Tropical es considerado uno de los ecosistemas más amenazados en el neotrópico (Janzen 1983). Debido a la fertilidad de sus suelos es lugar de desarrollo de poblaciones humanas y objeto de una intensa transformación (Janzen 1983; Ceballos 1995). Los bosques secos, a pesar de albergar una gran biodiversidad, que en algunos casos es única, son los ecosistemas menos estudiados y uno de los más amenazados (Janzen 1983; WRI 2001; Laval \& Bernal 2002); sólo un 5\% de éstos está protegido (WRI 2001). El reconocimiento y monitoreo de la biodiversidad en un área en particular nos permite documentar el estado de conservación en el que se encuentran las comunidades biológicas de un ecosis-
Durán, A. A. y Pérez, S. C. 2015. Bat assemblages (Mammalia: Chiroptera) in two areas of the Department of Sucre, Colombia. Acta Zoológica Mexicana (n. s.), 31(3): 358-366.

ABSTRACT. The objective of this study was to determine the structure and composition of two bat assemblages around the weather station Primates and "La Flojera" farm, in the department of Sucre, Colombia, from June 2011 to March 2012. The capture of bats was performed using mist nets, active from 18:00 to 24:00 h. We captured 427 bats belonging to 26 species, 17 genera, 6 subfamilies and 4 families. The family Phyllostomidae was the most diverse with 20 species. Of all caught individuals $97.9 \%$ belonged to the Phyllostomidae family. Artibeus planirostris was the most abundant with 139 individuals followed by, in decreasing order, Phyllostomus discolor with 56, Desmodus rotundus and Sturnira lilium with 37 individuals. According to the estimates of richness the sampling efficiency ranged between 80 and $90 \%$. The most common trophic guild was the frugivorous guild (42.3\%). The Shannon-Wiener diversity index was 2.40 in Primates and 2.0 in "La Flojera" farm. The species dominance was the highest (85\%) in Primates Station. The similarity between the bat communities based on the index of Jaccard was 0.53 . The differences found between the two bats communities can be related to differences in vegetation type at each locality.

Keywords: Artibeus planirostris, assemblages, bats, Sucre.

tema, además de ser el paso inicial para cualquier plan de manejo de los recursos naturales (Castro-Luna et al. 2007).

Los murciélagos se constituyen como uno de los grupos más importantes en la evaluación del impacto de la fragmentación sobre la diversidad del paisaje, debido a que forman un grupo abundante, diverso y fácil de muestrear (Jones et al. 2009). Sin embargo, aunque tienen la capacidad de volar y por consiguiente, de acceder a diferentes tipos de hábitats, la fragmentación y destrucción de sus ambientes, han alterado significativamente la riqueza y abundancia de los ensamblajes (Sánchez \& López 1998; Tirira 1998).

Debido a que estos mamíferos agrupan una gran variedad de gremios tróficos y al estar ampliamente distri- 
buidos en el Neotrópico (Tirira 1998), fueron calificados como bioindicadores de la calidad del hábitat (Heithaus et al. 1975). Además cumplen importantes funciones ecológicas, como dispersores de semillas, polinizadores de flores y como controladores eficaces de algunos insectos que actúan como plagas (Heithaus et al. 1975; Castro-Luna et al. 2007).

En Colombia se encuentran nueve familias de murciélagos: Emballonuridae, Noctilionidae, Mormoopide, Phyllostomidae, Natalidae, Furipteridae, Thyropteridae, Molossidae y Vespertilionidae, con 198 especies, que representan más del 20\% de las especies en el mundo (Solari et al. 2013; Simmons 2005). Para el departamento Sucre están reportadas 56 especies de murciélagos (Sampedro et al. 2007; Galván et al. 2009; Cabrera 2011; Montes et al. 2012), aunque la gran diversidad de microhábitats, características climáticas, variedad de recursos tróficos, así como pocos trabajos mastofaunísticos en el área, nos permiten suponer que puedan presentarse nuevos registros. Por consiguiente, el objetivo de este trabajo es contribuir al conocimiento de la riqueza y abundancia de los murciélagos en el departamento Sucre, y comparar la composición específica en las dos zonas de estudio seleccionadas con diferentes grados de perturbación antrópica.

\section{MATERIALES Y MÉTODOS}

Área de estudio. El departamento Sucre se encuentra localizado en la planicie costera Caribe al norte de las cordilleras central y occidental, entre los $10^{\circ} 9^{\prime}$ y los $8^{\circ} 17^{\prime}$ $\mathrm{N}$ y los $74^{\circ} 32^{\prime}$ y $75^{\circ} 42^{\prime} \mathrm{O}$, con una superficie de 10.364 $\mathrm{km}^{2}$. La jurisdicción de la Corporación Autónoma Regional del departamento Sucre-CARSUCRE cubre la parte norte del Departamento, presentando una faja de colinas, que corresponden a la Serranía de San Jacinto o Montes de María. Entre estas colinas y la depresión del río San Jorge se localizan sabanas antrópicas de topografía ondulada (Aguilera 2005). El trabajo se llevó a cabo en dos zonas del departamento Sucre pertenecientes a la subregión de los Montes de María; las zonas se encuentran dentro de los municipios de Colosó y Sincelejo (Fig. 1).

ZONA I: Corresponde al fragmento de bosque seco que se encuentra en la inmediaciones de la Estación Meteorológica Primates, de la Corporación Autónoma Regional del departamento Sucre-CARSUCRE (municipio de Colosó), localizada a los $9^{\circ} 31^{\prime} 47.15^{\prime}$ ' $\mathrm{N}$ y los $75^{\circ}$ 21'5.80'” O, en las estribaciones de la Serranía de Coraza. La zona se caracteriza por presentar bosques higrotropofíticos, subhigrofíticos y freatofíticos. Los elementos flo-

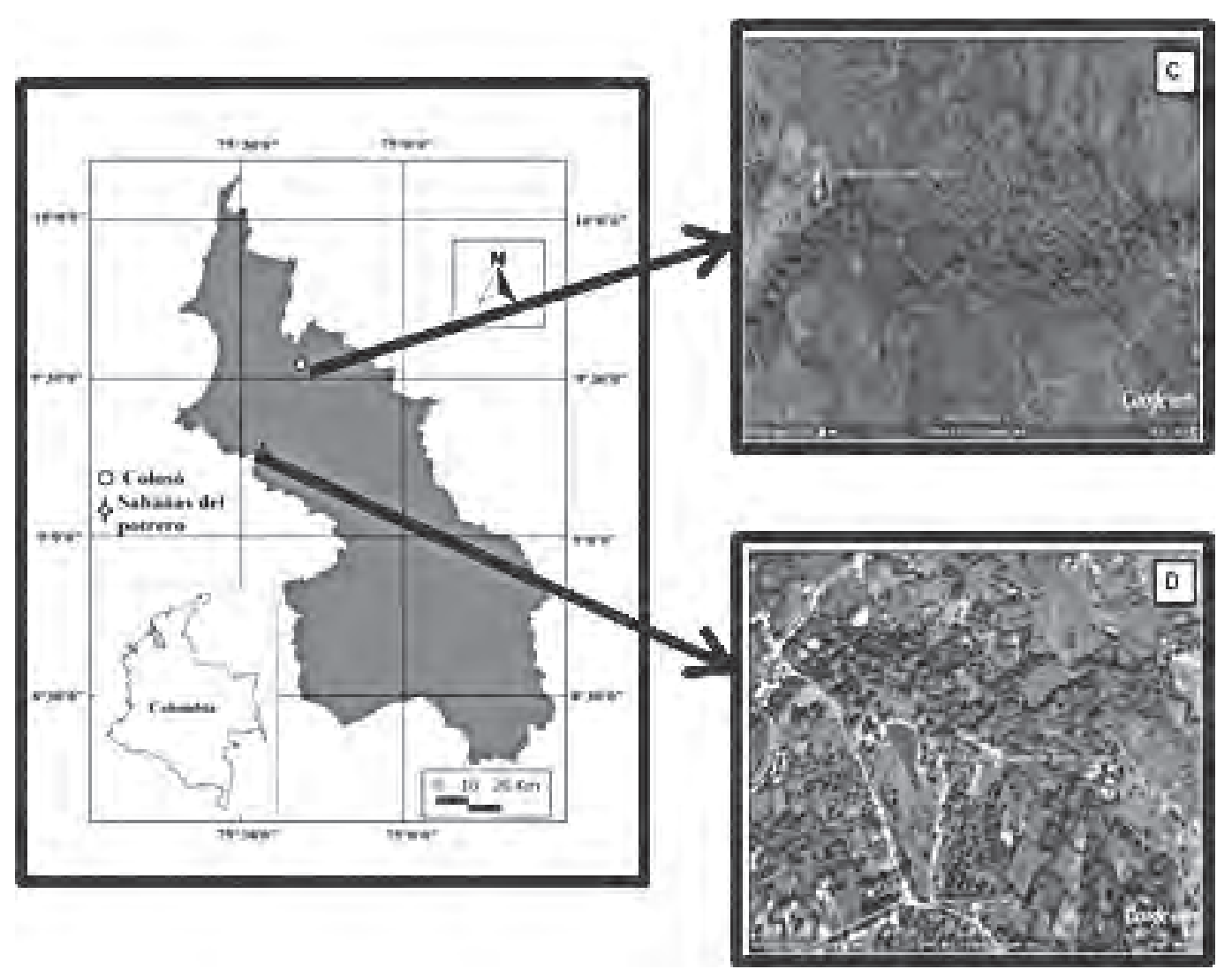

Figura 1. Ubicación geográfica de las zonas de estudio. A: Colombia. B: Sucre. C: Estación Primates. D: Finca La Flojera “El Palmar”. 
rísticos principales de la zona son: Anacardium excelsum (caracolí), Brownea macrophylla (arizal), Enterolobium cyclocarpum (orejero), Bursera simaruba (indio encuero), Pachira quinata (ceiba tolua), Manilkara zapota (níspero), Pseudobombax septenatum (majagua), Uribea tamarindoides (tamarindo de mico), Hura crepitans (ceiba de leche), Sterculia apetala (camajón), y Spondias mombin (jobo), entre otras (Cuervo et al. 1986). Presenta un clima cálido seco, correspondiente en la clasificación de Holdridge (1979) a bosque seco tropical (bs-T) con una altura de $300 \mathrm{msnm}$, una temperatura media de 27 ${ }^{\circ} \mathrm{C}$ y una pluviometría que alcanza los $1200 \mathrm{~mm}$ anuales (Domínguez et al. 2011).

ZONA II: Ubicado en la finca La Flojera, corregimiento de Sabanas del Potrero, Sincelejo, el sitio se encuentra localizado a 9 $14^{\prime}$ 50.5' ' $\mathrm{N}$ y a los $75^{\circ} 26^{\prime}$ 01.2'” O; con una temperatura media anual de $27^{\circ} \mathrm{C}$ y una precipitación anual que alcanza los $1200 \mathrm{~mm}$ (IGAC 1969). En esta zona predomina Sabal mauritiiformis (palma amarga). El área de estudio tiene una extensión aproximada de 18 hectáreas, en donde aproximadamente el $70 \%$ se encuentra representado por cobertura de Palma Amarga (S. mauritiiformis) y el 30\% restantes está compuesto de especie vegetales como Cedrela odorata (cedro), Mangifera indica (mango), Anacardium excelsum (caracolí), Albizia niopoides (guacamayo), Guazuma ulmifolia (guácimo), Piptadenia pitteri (carbonero), Astronium graveolens (santa cruz), Manilkara zapota (níspero), Cordia alliodora (vara de humo) entre otros. Las actividades que se realizan en la finca son la ganadería, piscicultura y agricultura (cultivos de maíz, yuca, ñame y plátano). Además, se practica la crianza de aves de corral (pavos y gallinas) y porcinos.

Trabajo de campo. Para cada zona de estudio se utilizaron tres redes de niebla $(12 \times 2.5 \mathrm{~m})$ ubicadas a nivel del suelo (0-3 m), que permanecieron abiertas durante tres noches consecutivas, desde las 18:00 hasta las 24:00 horas. Se realizaron seis muestreos en cada sitio de estudio durante los meses de junio de 2011 a marzo de 2012, totalizándose 12 muestreos. Cada red estuvo separada de la otra por una distancia mínima de $50 \mathrm{~m}$, con el fin de maximizar el éxito de captura. Mientras las redes estuvieron activas, se revisaron cada 30-45 minutos y los murciélagos capturados fueron depositados en bolsas de tela para identificarlos posteriormente mediante claves de campo de Muñoz (2001) y Linares (2000). De cada ejemplar recolectado se tomaron las siguientes medidas morfométricas, útiles para la determinación taxonómica: longitud del antebrazo (AB), longitud de la pata (LP), longitud de la oreja (LO), longitud del trago (LTr), longitud total (LT) y envergadura alar. Las medidas fueron tomadas con un calibrador vernier con precisión de 0,05 mm. Una vez tomadas las medidas e identificados taxonómicamente, los murciélagos fueron liberados en el sitio de captura. El arreglo taxonómico siguió a Solari et al. (2013). Los ejemplares que no pudieron ser identificados con certeza en campo, fueron sacrificados mediante inyección torácica de Xilocaína al 2\% y luego se preservaron en frascos con etanol al 70\%. La identificación fue confirmada por el profesor Jesús Ballesteros (Universidad de Córdoba) y los ejemplares que se conservan como vouchers fueron depositados en la Colección de Zoología de la Universidad de Córdoba (Montería, Córdoba-Colombia; CZUC), con número de referencia CZUC 557-609.

Análisis de datos. Se determinó el esfuerzo de muestreo, tomado como: $\mathrm{E}_{\mathrm{m}}=\mathrm{m}^{2}$ red $\times$ horas de exposición y el éxito de captura, mediante el número de individuos capturados dividido por el esfuerzo de muestreo y multiplicado por 100. La representatividad del muestreo se evaluó a partir de curvas de acumulación, mediante el uso del estimador de riqueza de Jack-knife 1 Mean, el cual se considera apropiado para organismos móviles (Colwell 2006; Brose et al. 2010), así como el de Jack-knife 2 Mean, mediante el cual se estima el número posible de especies encontradas, si el esfuerzo de muestreo fuese más prolongado (Roncancio \& Estévez 2007); estas estimaciones se hicieron con el programa EstimateS 8.2 (Colwell 2006). Para la riqueza y abundancia se emplearon los índices de Shannon-Wienner y el de equidad de Pielou (Moreno \& Halffter 2001). Además, para comparar las zonas de estudio se utilizó el índice de similitud cualitativo de Jaccard donde el intervalo de valores para este índice va de 0 cuando no hay especies compartidas entre ambos sitios, hasta 1 cuando los dos sitios tienen la misma composición de especies.

\section{RESULTADOS}

El esfuerzo de muestreo fue de $19,440 \mathrm{~m}^{2}$ red $\times$ hora $\mathrm{y}$ con un éxito de captura de 2.20 individuos hora/red. Se capturaron 427 murciélagos pertenecientes a 26 especies, 17 géneros, 6 subfamilias y 4 familias. La familia Phyllostomidae fue la más diversa en especies (20 especies registradas) y la más abundante (418 individuos, es decir, el 97.9\% de todas las capturas). Otras familias representadas en el estudio fueron Emballonuridae (2 especies y 5 individuos), Mormoopidae (3 especies y 3 individuos) y Noctilionidae (1 especie y 1 individuo; Cuadro 1).

En el ensamblaje de la finca "La Flojera" se capturaron 177 individuos y las especies más abundantes fueron, Artibeus jamaicensis, A. lituratus, A. planirostris y $P$. discolor. Para el ensamblaje de "Primates", las especies que concentraron la mayoría de las capturas fueron $A$. 
Cuadro 1. Riqueza y abundancia por especies, de murciélagos registrados en el departamento Sucre. GRE: gremio trófico, I = insectívoro, $\mathrm{H}$ = hematófago, $\mathrm{F}=$ frugívoro, $\mathrm{NP}=$ néctar-polinívoro, $\mathrm{O}=$ omnívoro, Car = carnívoro, $\mathrm{P}$ = piscívoro, FJ = finca "La Flojera”, $\mathrm{PRI}=$ Estación Primates, $\mathrm{Ab}=$ Abundancia absoluta, $\mathrm{Ab} \%=$ Abundancia relativa.

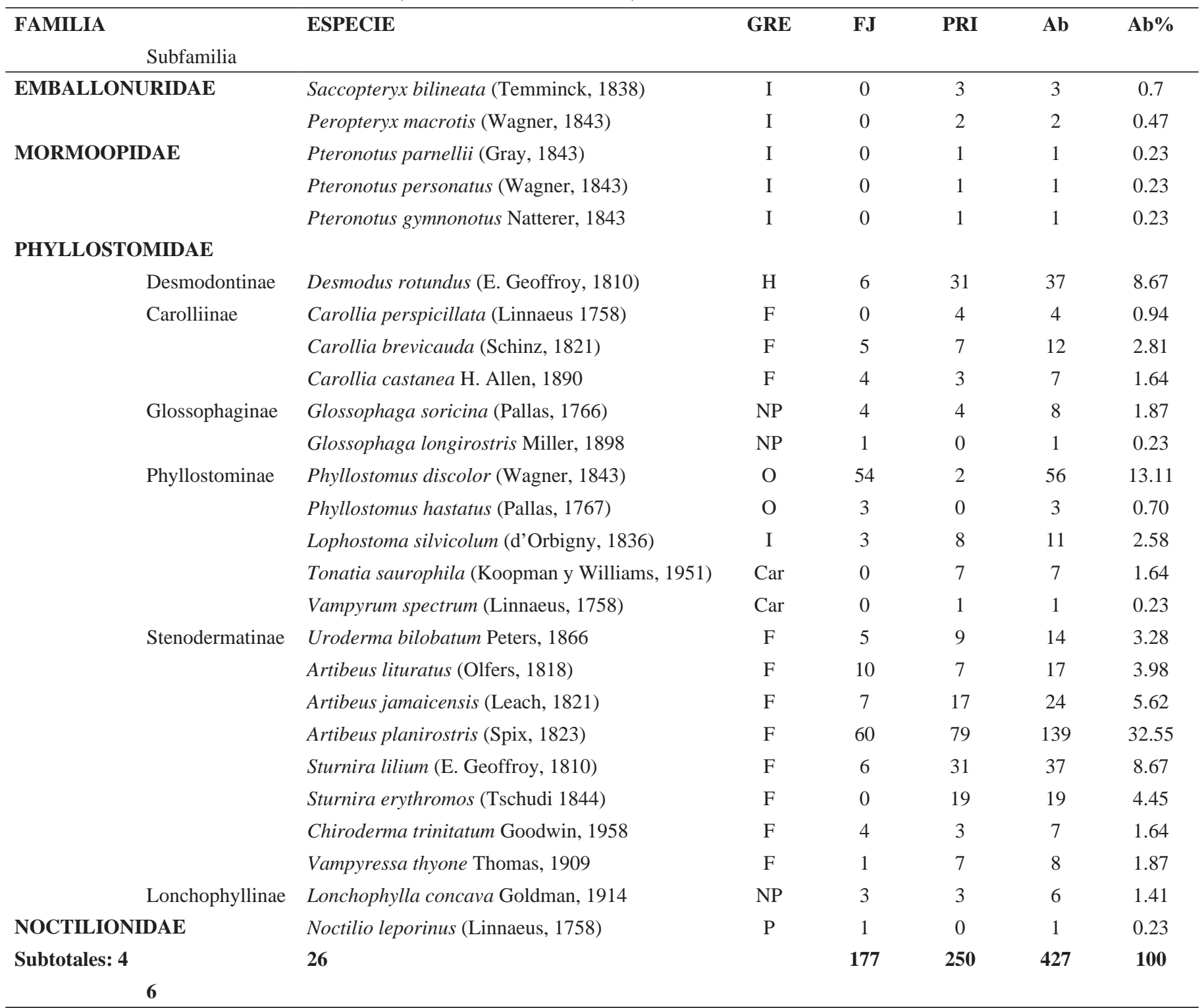

planirostris, Desmodus rotundus, Sturnira erythromos y S. lilium, agrupando a 250 individuos del total capturado (427).

Las especies A. planirostris 32.5\% ( $\mathrm{n}=139), P$. discolor $13 \%(\mathrm{n}=56)$, S. lilium y D. rotundus con un $8.6 \%$ ( $\mathrm{n}=37$ ) cada uno, presentaron las mayores abundancias relativas respecto al total de los individuos capturados durante los muestreos realizados, mientras que las especies Glossophaga longirostris, Noctilio leporinus, Pteronotus gimnonotus, P. parnellii, P. personatus y Vampyrum spectrum presentaron abundancia baja, con sólo un individuo capturado para cada especie (Cuadro 1 y Fig. 2).

Con los estimadores de riqueza no paramétricos utilizados para dibujar las curvas de acumulación (Figs. 3 y 4) se obtuvo una eficiencia de muestreo del $84 \%$ en "Primates" y del 87\% para la Finca "La Flojera”, lo cual refleja una buena representatividad de los sitios muestreados.

El valor del índice de diversidad de Shannon-Wiener fue de 2.0 para la finca "La Flojera" y de 2.4 para el fragmento de bosque seco de la Estación Primates. El valor de la equidad fue de 0.706 y 0.765 respectivamente. El resultado de la prueba $t$ modificada por Hutcheson $\left(t_{0.05}\right.$ (2)365 $=1.96)$ indica que se pueden establecer diferencias estadísticamente significativas en la diversidad de los ensamblajes de ambas zonas de estudio. Por otro lado, la comparación de los ensamblajes con el índice de Jaccard cualitativo arrojó un valor de similitud de 0.53 lo cual su- 


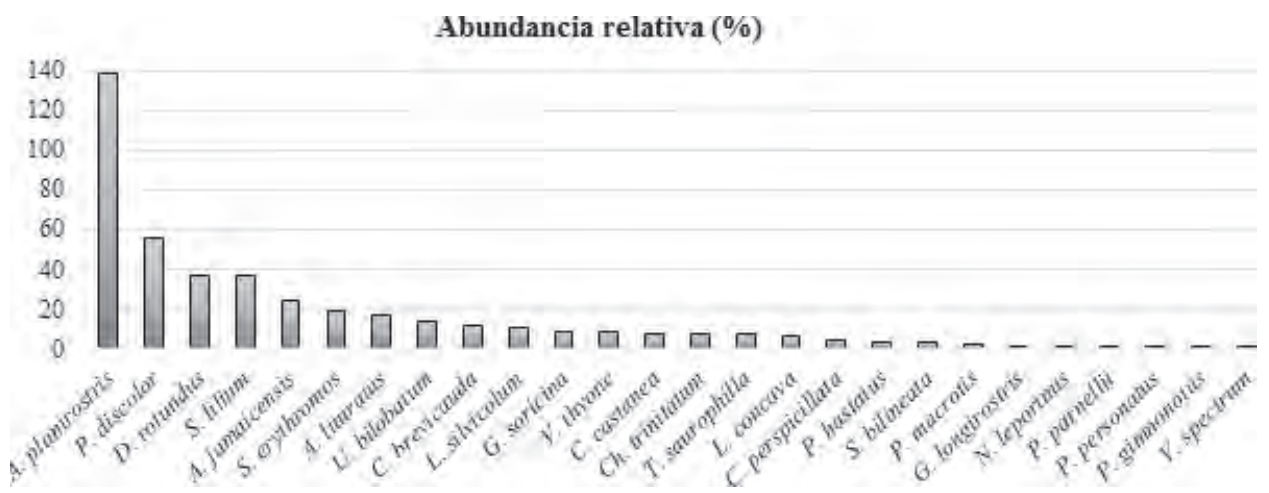

Figura 2. Abundancia relativa de los murciélagos registrados en dos zonas del departamento Sucre, Colombia.

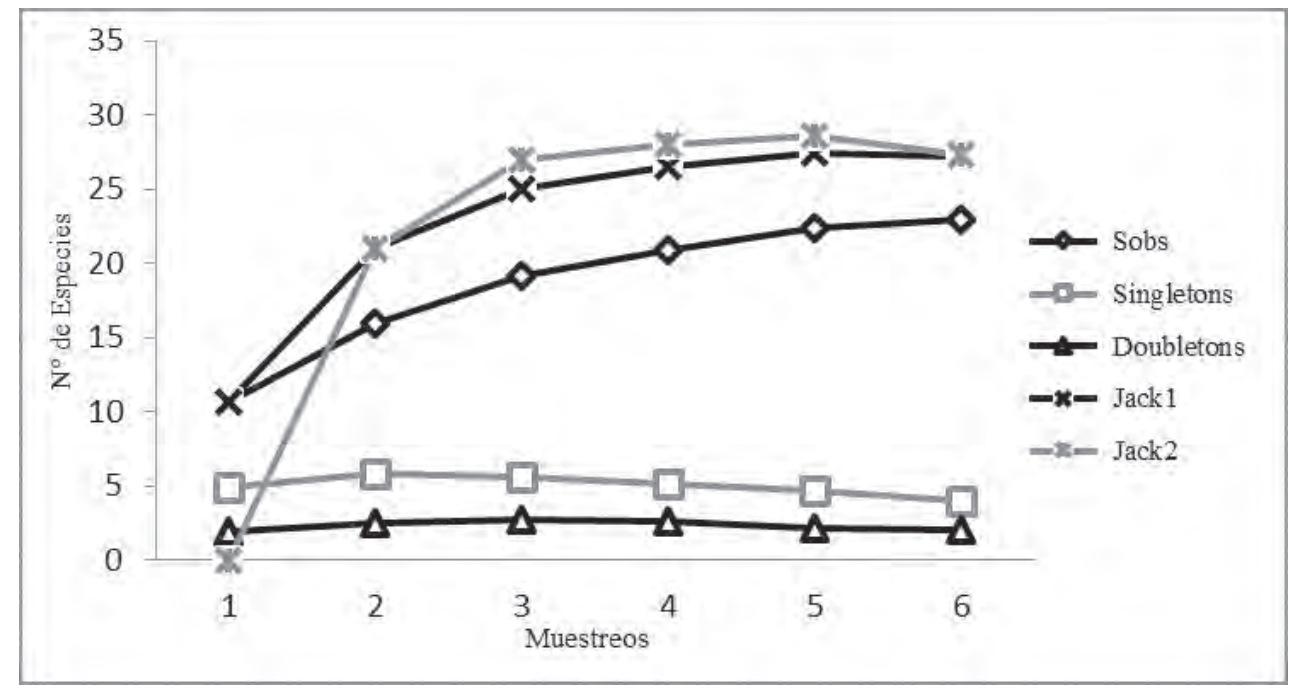

Figura 3. Curva de acumulación de especies de murciélagos capturados con redes de niebla en la Estación Primates, Colosó, Sucre.

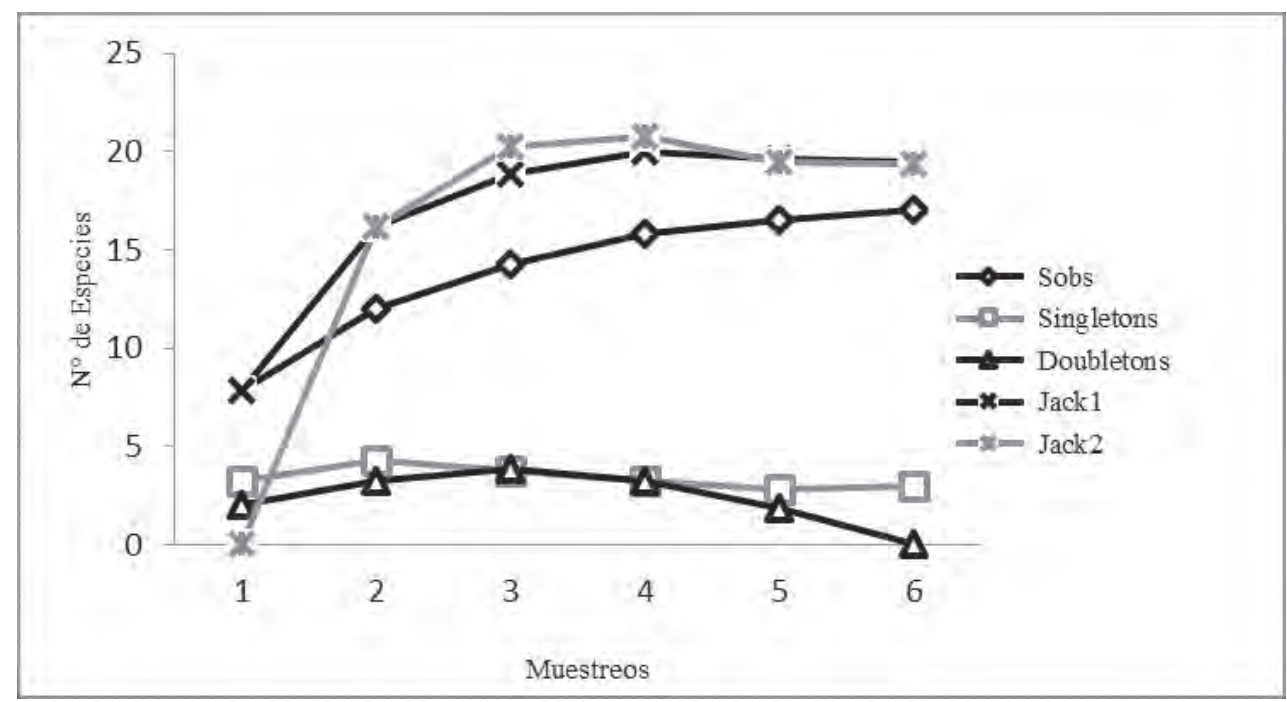

Figura 4. Curva de acumulación de especies de murciélagos capturados con redes de niebla en finca “La Flojera”, Sincelejo, Sucre. 
giere que los ensamblajes son medianamente diferentes en la composición de las especies.

En cuanto a la estructura trófica, en general el gremio de los frugívoros fue el más abundante, representado por 11 especies, incluyendo a tres especies de los géneros Artibeus, Sturnira (subfamilia Stenodermatinae) y tres especies del género Carollia (subfamilia Carollinae) que pertenecen a la familia Phyllostomidae, la cual constituyó el $42.3 \%$ del total de los individuos capturados. El segundo gremio trófico más importante fue el de los insectívoros, con seis especies y el $23 \%$ de las capturas, seguido por los néctar-polinívoros (tres especies y el 11.5\%). Los omnívoros y carnívoros (dos especies y el 7.6\%), y los hematófagos y piscívoros (una especie cada uno y el 3.85\%) fueron los gremios menos abundantes (Cuadro 1 y Fig. 5).

Para el caso de la finca "La Flojera” y Primates, el gremio de los frugívoros tuvo una mayor abundancia (52.9\% y $47.8 \%$, respectivamente) seguido del gremio de los insectívoros donde fue mayor en Primates (26\%) y en la finca "La Flojera" (5.8\%); los néctar-polinívoros estuvieron representados con el $\mathbf{1 7 . 6 \%}$ en la finca "La Flojera" y el 8.6\% en Primates; el gremio de los carnívoros estuvo ausente en la finca "La Flojera", mientras que los piscívoros estuvo ausente en Primates (Fig. 5).

Las especies compartidas para las dos zonas de estudio fueron Artibeus jamaicensis, A. lituratus, A. planirostris, C. brevicauda, C. castanea, Chiroderma trinitatum, $D$. rotundus, L. concava, P. discolor, S. lilium, U. bilobatum, G. soricina y Vampyressa thyone. Las especies únicas para el cultivo de palma amarga de la finca La Flojera fueron Glossophaga longirostris, Phyllostomus hastatus y $N$. leporinus y para la estación Primates las especies del género Pteronotus ( $P$. personatus, $P$. parnellii, $P$. gymnonotus), Saccopterix bilineata, Peropterix macrotis, $C$. perspicillata, Tonatia saurophila, Vampyrum spectrum y Sturnira erythromus (Cuadro 1).

\section{DISCUSIÓN}

Las 26 especies de murciélagos distribuidas en 17 géneros y cuatro familias registradas para la finca "La Flojera" y la Estación Primates, corresponden al 13.13\% de las 198 especies reportadas para el país (Solari et al. 2013) y al 46.4\% de las 56 especies reportadas para el departamento de Sucre (Montes et al. 2012; Cabrera 2011; Galván et al. 2009; Sampedro et al. 2007) Además, se registraron cinco nuevas especies para el departamento: Chiroderma trinitatum, Glossophaga longirostris, Lophostoma silvicolum, Tonatia saurophila y Vampyressa thyone.

La familia Phyllostomidae es considerada la más abundante en el neotrópico, debido a su amplio rango de recursos alimenticios y su gran capacidad adaptativa (Fleming 1986; Muñoz 1986; Ospina \& Gómez 1999; Freeman 2000). De este modo, no es raro que las especies pertenecientes a Phyllostomidae fueran las más abundantes en este estudio, y también en Colombia con 118 especies (Mantilla-Meluk et al. 2009), siendo estos resultados consistentes con otros estudios realizados en bosque seco tropical (Sánchez \& López 1998; Stoner 2005; Vela \& Pérez 2012).

Según Galindo (1998) la fragmentación del hábitat puede ser un factor importante que determina que las especies de murciélagos visiten las áreas perturbadas y, consecuentemente, puede influir en la composición y estructura de la vegetación establecida en dichos terrenos. Es muy factible que la subfamilia Stenodermatinae sea la más abundante en los sitios perturbados (Stoner 2005)

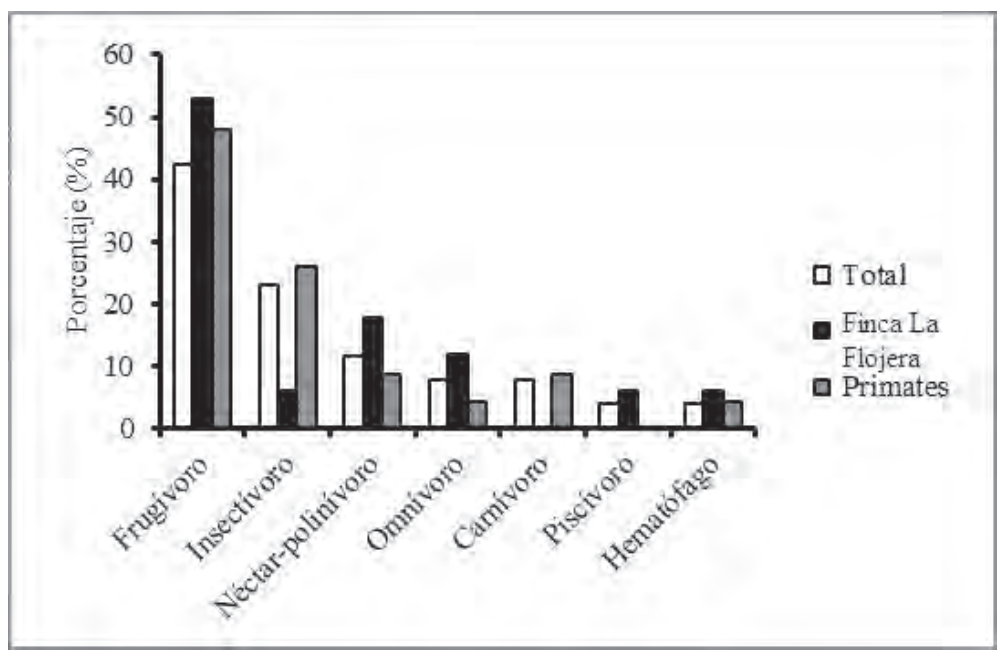

Figura 5. Estructura trófica de los ensamblajes de murciélagos presentes en dos zonas del departamento. 
debido a que sus representantes dispersan semillas de manera muy eficiente (Galindo 1998); probablemente la regeneración de la vegetación en los potreros dependerá, en gran medida, de la presencia de estos filostómidos. Por consiguiente, la abundancia y diversidad de especies registradas, muestran que el ensamblaje de murciélagos está fuertemente dominado por algunas especies de la subfamilia Stenodermatinae como A. jamaicensis, $A$. planirostis, C. brevicauda, C. perspicillata, D. rotundus, $S$. lilium, las cuales podrían reflejar la perturbación antrópica (Coutinho \& Bernard 2012) que se evidenció en las zonas de estudio, siendo esto concordante con otros estudios en ambientes fragmentados (Moreno \& Halfter 2001; Montiel et al. 2006).

Estudios realizados en la estación Primates, por Galván et al. (2009), Cabrera (2011) y Montes et al. (2012), registran una riqueza de murciélagos de 27, 19 y 17 especies respectivamente, esto es similar a lo encontrado en este estudio donde se registran 23 especies para un total de 44 reportadas para el sitio de estudio.

Aunque la representatividad del muestreo en las dos zonas de estudio fue alta (entre un 84 y $87 \%$ de las especies esperadas), las especies de las familias Molossidae y Vespertilionidae no fueron registradas, a pesar de haber sido reportadas para el departamento (Sampedro et al. 2007; Galván et al. 2009). No cabe duda que el método de captura (redes de niebla a nivel de sotobosque) influyó para que no fuera posible registrar estas familias, ya que sus hábitos de vuelo y forrajeo son a nivel del dosel del bosque (Fleming et al. 1972). Otro factor que pudo afectar la representatividad, fue el período de muestreo realizado entre las 18:00 hasta 24:00 horas, y que, por lo tanto, excluyó aquellos murciélagos cuya actividad la realizaban en las horas de la madrugada.

En la estación Primates y la finca "La Flojera” el valor $H^{\prime}$ fue de 2.4 y 2.0 respectivamente. La diferencia es estadísticamente significativa, lo cual podría deberse a la disponibilidad de recursos que se pueden encontrar en la finca, acompañado de que las especies encontradas en esta zona de estudio (Cuadro 1) son bastante adaptables y propias de sitios perturbados (Fleming 1986).

Por otro lado, la similitud entre las dos zonas de estudio fue de 0.53 , es decir, el $53 \%$, este valor es bajo según Sánchez \& López (1998) quienes establecen que dos grupos faunísticos son similares cuando sobrepasan un $66,6 \%$ de similitud. De acuerdo a esto, y con las reservas de haber comparado solo una repetición de cada ambiente, se puede inferir que los ensamblajes de los dos ambientes (áreas) de estudio, no son similares, aunque comparten algunas especies. Esto posiblemente se deba a las diferencias en la vegetación de cada una de las zonas de muestreo y en su grado de perturbación.
La dominancia de las especies A. planirostris y $P$. discolor en las zonas en las que se realizó el trabajo de campo de esta investigación, es consistente con los trabajos de Medellín et al. (2000) y Ballesteros \& Racero (2012), los cuales consideran que estas especies presentan hábitos generalistas en cuanto a dieta y uso de hábitat, por lo tanto se presentan en altos números. En estudios realizados por Passos \& Graciolli (2004) y Morim \& Nobre (2009), se reconoce la importancia de los géneros Cecropia, Piper y Solanum en la dieta de algunas especies de murciélagos como A. lituratus, A. jamaicensis, C. brevicauda y Sturnira lilium. Aunque en este estudio no se realizó una caracterización florística, sí se observó la presencia de los géneros Cecropia, Piper y Solanum, en las zonas aledañas al área de estudio. Además, Jurado et al. (2010), registraron la presencia de estos géneros de plantas en la estación Primates; al igual que Rodríguez (2012), quien los reporta en bosques secos de los departamentos de Atlántico y Bolívar. Lo anterior podría explicar la presencia de estas especies de murciélagos en las zonas de estudio.

Respecto a la composición trófica de las especies, se esperaba que el gremio de los hematófagos fuera el más dominante debido a la marcada presencia de ganado vacuno en las áreas en las que se desarrolló el estudio. Consistente con esta predicción, el vampiro fue la tercera especie, junto con Sturnira lilium, más abundante. Lo anterior contrasta con la investigación realizada por Calonge et al. (2010) en una finca ganadera, la cual informó de la dominancia de los frugívoros, y de solo dos capturas de hematófagos.

Los resultados encontrados en cuanto a composición de especies, indica que hay una mayor riqueza en los fragmentos boscosos más conservados, como ocurrió en la estación Primates lo cual puede significar que el bosque de la estación provee de refugios y alimentos en una vasta extensión, entre arroyos y sistemas montañosos. Mientras que la finca "La Flojera" es el único sitio forestado que existe en el corregimiento de Sabanas del Potrero y se presenta como un parche de bosque constituido por palma amarga, que ofrece refugio y alimento a los murciélagos en una zona donde la deforestación del bosque seco tropical para la creación de zonas de pastoreo de ganado vacuno, es muy evidente. Por esta razón, es importante impulsar y ejecutar acciones de conservación encaminadas a la protección de los cultivos de palma amarga y de los remanentes de bosque secos que aún quedan en el departamento Sucre. Si se sigue con la conducta de reducir el área de la cobertura arbórea para la implantación de pasturas dirigida a la ganadería extensiva y la agricultura en zonas onduladas, esto repercutirá en la desaparición de las especies vegetales aún presentes, las que ofrecen refugio y alimento a los murciélagos y otros animales que 
aún habitan las zonas que fueron objeto de la presente investigación.

AGRADECIMIENTOS. Al profesor Jesús Ballesteros Correa (Universidad de Córdoba), por su incondicional ayuda en la identificación taxonómica de los murciélagos; a Alejandro Zamora, jefe del departamento de fauna de la Corporación Autónoma Regional de SucreCARSUCRE, por facilitarnos los materiales de campo y el permiso para quedarnos en las instalaciones de la Estación Meteorológica Primates. A los señores Pedro Márquez y Cristóbal por su compañía y colaboración durante nuestra estadía en la Estación. Al señor Antonio “Toño" Mendoza y a su familia por acogernos durante el tiempo que trabajamos en la Finca "La Flojera". Damos gracias a nuestros amigos e integrantes del Grupo de Investigación en Zoología y Ecología de la Universidad de Sucre-GIZEUS por todo el apoyo en este trabajo.

\section{LITERATURA CITADA}

Aguilera, M. 2005. La Economía del Departamento de Sucre: Ganadería y Sector Público. Documentos de Trabajo sobre Economía Regional N 63, Banco de la República. Centro de Estudios Económicos Regionales, Cartagena. 126 pp.

Ballesteros, J. \& Racero. J. 2012. Murciélagos del área urbana en la ciudad de Montería, Córdoba, Colombia. Revista MVZ Córdoba, 17: 3193-3199.

Brose, U., Martinez, N. \& Williams, R. 2010. Estimating species richness: sensitivity to sample coverage and insensitivity to spatial patterns. Ecology, 84: 2364-2377.

Cabrera, Y. 2011. Composición, estructura y distribución del ensamble de murciélagos durante la época de lluvia presentes en el departamento de Sucre-Colombia. Trabajo de grado. Santa Marta D.T.C.H. Universidad del Magdalena. Facultad de Ciencias Básicas. Programa de Biología. 67 p.

Calonge, B., Vela, M. \& Pérez, J. 2010. Murciélagos asociados a una finca ganadera en Córdoba (Colombia). Revista MVZ Córdoba, 15: 1938-1943

Castro-Luna, A., Sosa, V. J. \& Castillo-Campos, G. 2007. Bat diversity and abundance associated with the degree of secondary succession in a tropical forest mosaic in southeastern Mexico. Animal Conservation, 10: 219-229.

Ceballos, G. 1995. Vertebrate diversity, ecology, and conservation in neotropical dry forest. pp. 195-222. In: Bullock, S., Medina, E. \& Mooney, H. A. (Eds.). Tropical deciduous Forest Ecosystem. Cambridge University Press, Cambridge.

Colwell, R. 2006. EstimateS: statistical estimation of species richness and shared species from samples. Version 8.2. Department of Ecology and Evolutionary Biology, University of Connecticut, U.S.A. Persistent URLpurl.oclc.org/estimates.

Coutinho, C. \& Bernard, E. 2012. Neotropical bats as indicators of environmental disturbance: what is the emerging message? Acta Chiropterologica, 14: 143-151.

Cuervo, A., Barbosa, C. \& de la Ossa, V. J. 1986. Aspectos ecológicos y etológicos de primates con énfasis en Alouatta seniculus (Cebidae), de la región de Colosó, Serranía de San Jacinto (Sucre), Costa Norte de Colombia. Caldasia, 14: 709-742.

Domínguez, K., González, J. \& Támara, Y. 2011. Composición y abundancia de Mariposas diurnas (Lepidoptera: Rhopalocera) en la vegetación de la ribera del arroyo el sereno, municipio de Colo- só, Departamento de Sucre. Trabajo de grado. Sincelejo. Universidad de Sucre. Facultad de Educación y Ciencias. Departamento de Biología. 130 pp.

Fleming, T. 1986. The structure of neotropical bat communities: a preliminary analysis. Revista Chilena de Historia Natural, 59: $135-150$.

Fleming, T., Hooper, E. \& Wilson, D. 1972. Three Central American bat communities: structure, reproductive cycles, and movement patterns. Ecology, 53: 555-569.

Freeman, P. 2000. Macroevolution in microchiropteran: recoupling morphology and ecology with phylogeny. Evolutionary Ecology Research, 2: 317-335.

Galindo, J. 1998. Dispersión de semillas por murciélagos: su importancia en la conservación y regeneración del bosque tropical. Acta Zoológica Mexicana (n. s.), 73: 57-74.

Galván, S., Galván-Guevara, S., Sierra, M. I., Gómez, F. H., De La Ossa, V. J. \& Fajardo-Patiño, A. 2009. Biodiversidad en el área de influencia de la estación Primates de Colosó, Sucre, Colombia. Revista Colombiana de Ciencia Animal, 1: 98-121.

Heithaus, E. R., Fleming, T. H. \& Opler, P. W. 1975. Foraging patterns and resource utilization in seven species of bats in a seasonal tropical forest. Ecology, 56: 841-854.

Holdridge, L. 1979. Ecología basada en zonas de vida. Instituto Interamericano de Ciencias Agrícolas, San José, Editorial IICA (Costa Rica). 375 pp.

IGAC. 1969. Monografía del Departamento de Sucre. Instituto Geográfico Agustín Codazzi. Bogotá, Colombia.

Janzen, D. 1983. Dispersal of seeds by vertebrate guts. pp. 232-262 In: Futuyama, D. J. \& Slatkin, M. (Eds.). Coevolution. Sinauer Associates Inc. Sunderland.

Jones, G., Jacobs, D, Kunz, T., Willigs, M. \& Racey, P.A. 2009. Carpe noctem: the importance of bats as bioindicators. Endangered Species Research, 8: 93-115.

Jurado, H., Martínez, C. \& González, J. C. 2010. Composición de especies y distribución espacial de la avifauna asociada a la microcuenca del arroyo el sereno, reserva forestal protectora serranía de coraza y montes de maría, Colosó (Sucre-Colombia). Trabajo de grado de Biología. Sincelejo. Universidad de Sucre. Facultad de educación y ciencias. Programa de Biología, 118 pp.

Laval, R. \& Bernal, R. 2002. Murciélagos de Costa Rica. $1^{\text {a }}$ ed. Santo Domingo de Heredia, Costa Rica: Instituto Nacional de Biodiversidad, INBIO. 320 pp.

Linares, O. 2000. Murciélagos. Mamíferos de Venezuela. pp. 349591. Linares. O. (Ed.). Sociedad Conservacionista Audubon de Venezuela. Venezuela.

Mantilla-Meluk, H., Jiménez, A. \& Baker, R. 2009. Phyllostomid Bats of Colombia: Annotated Checklist, Distribution, and Biogeography. Special Publications Museum of Texas Tech University, 56: 1-37.

Medellín, R., Equihua, M. \& Amín, M. 2000. Bat diversity and abundance as indicators of disturbance in Neotropical rainforests. Conservation Biology, 14: 1666-1675.

Montes, G., Durán, A., López, Y., Díaz, J. \& Oviedo, N. 2012. Nuevos datos sobre la distribución de Pteronotus personatus (Wagner, 1843) (Chiroptera: Mormoopidae) en Colombia. Revista Colombiana de Ciencia Animal, 4: 435-440.

Montiel, S., Estrada, A. \& León, P. 2006. Bat assemblages in a naturally fragmented ecosystem in the Yucatan Peninsula, Mexico: species richness, diversity and spatio-temporal dynamics. Journal of Tropical Ecology, 22: 267-276.

Moreno, C. \& Halffter, G. 2001. Spatial and temporal analysis of $\alpha$, 
$\beta$ and $\gamma$ diversities of bats in a fragmented landscape. Biodiversity and Conservation, 10: 367-382.

Morim, R. \& Nobre, C. 2009. Dieta de Artibeus lituratus (Olfers, 1818) em área urbana na cidade do Rio de Janeiro: frugivoria e novo registro de folivoria. Chiroptera Neotropical, 15: 487-493.

Muñoz, J. 1986. Murciélagos del Parque Natural El Refugio (Antioquia, Colombia). Actualidades Biológicas, 15: 26-34.

Muñoz, J. 2001. Los murciélagos de Colombia. Sistemática, distribución, descripción, historia natural y ecología. Editorial Universidad de Antioquia, Medellín. 391 pp.

Ospina, Y. \& Gómez, G. 1999. Riqueza, abundancia relativa y patrones de actividad temporal de la comunidad de murciélagos de la Reserva Natural La Planada, Nariño, Colombia. Revista de la Academia Colombiana de Ciencias Exactas, Físicas y Naturales. Vol. 23 (Suplemento especial): 659-669.

Passos, F. \& Graciolli, G. 2004. Observações da dieta de Artibeus lituratus (Olfers) (Chiroptera, Phyllostomidae) em duas áreas do sul do Brasil. Revista Brasileira de Zoología, 21: 487-489.

Rodríguez, G. 2012. Lista comentada de las plantas vasculares de bosques secos prioritarios para la conservación en los departamentos de Atlántico y Bolívar (Caribe colombiano). Biota Colombiana, 12: 7-39.

Roncancio, N. \& Estévez, J. 2007. Evaluación del ensamblaje de murciélagos en áreas sometidas a regeneración natural y a restauración por medio de plantaciones de Aliso. Boletín Científico de Museo de Historia Natural de la Universidad de Caldas, 11: 131-143.

Sampedro, A., Martínes, C. M., De La Ossa, K., Otero, Y. L., San- tos, L. M., Osorio, S. \& Mercado, A. M. 2007. Nuevos registros de especies de murciélagos para el Departamento de Sucre y algunos datos sobre su ecología en esta región colombiana. Caldasia, 29: 355-362.

Sánchez, O. \& López, G. 1988. A theoretical analysis of some indices of similarity as applied to biogeography. Folia Entomológica Mexicana, 75: 119-145.

Simmons, N. B. 2005. Order Chiroptera. pp: 312-529. In: Wilson, D. E. \& Reeder, D. M. (Eds.). Mammals species of the world: $a$ taxonomic and geographic reference. 3a Ed. The Johns Hopkins University Press. Baltimore, USA.

Solari, S., Muñoz-Saba, Y., Rodríguez-Mahecha, J. V., Defler, T. R., Ramírez-Chávez, H. E. \& Trujillo, F. 2013. Riqueza, endemismo y conservación de los mamíferos de Colombia. Mastozoología Neotropical, 20: 301-365.

Stoner, K. 2005. Phyllostomid Bat Community Structure and Abundance in Two Contrasting Tropical Dry Forests. Biotropica, 37: 591-599.

Tirira, D. 1998. Biología, sistemática y conservación de los mamíferos en Ecuador. Memorias, Publicación especial. Pontificia Universidad Católica del Ecuador. Quito, Ecuador.

Vela, I. \& Pérez, J. 2012. Murciélagos asociados a remanentes de bosque seco tropical en un sistema de ganadería extensiva (Colombia). Chiroptera Neotropical, 18: 1089-1100.

World Resources Institute (WRI). 2001. A Guide to World Resources 2000-2001: People and Ecosystems: The Fraying Web of Life. First edition. Washington. 276 pp. 